\title{
Acknowledgemet of referees
}

Agricultural and Food Science expresses its sincere thanks to the following referees for their constructive critical reviews of one or more manuscripts during the year 2011.

Alakukku, L.

Andresen, L.

Astover, A.

Baar, J.

Ballantine, D.

Blanco, M.

Blombäck, K.

Bothe, $\mathrm{H}$.

Brazaityte, A.

Bunce, J.

Bunter, K.

Chai, W.

Chantigny, $\mathrm{M}$.

Chardon, W.

Charlebois, D.

Collado, M.C.

Dagerskog, L.

Danesi, F.

De Cara, S.
Demeyer, D.

Dries, L.

Edwards-Jones, G.

Ertbjerg, P.

Feldmann, F.

Fitter , A.

Folta, K.

Frankow-Lindberg, B.

García-Viguera, C.

Gierus, M.

Gil, M.

Goicoechea, N.

Grout, B.

Guillen, F.

Gullstrand, J.

Hamel, C.

Hijikata, N.

Hofgaard, I.

Hogewoning, S.W. 
Hooker, J.

Huuskonen, A.

Hyvönen, L.

Hönow, R.

Jain, M.

Janouskova, M.

Jansa, J.

Jayaraj, J.

Johansson, B.

Johansson, E.

Johnson, N.

Joner, E.

Koeck, A.

Koltai, $\mathrm{H}$.

Kopmanis, J.

Kopp, K.

Lehtonen, $\mathrm{H}$.

Lloyd, T.

Macek, I.

Maobe, S.N.

Marshall, J.

Martinez Medina. A.
Martínez-Romero, D.

McKinnon, J.

Moore, D.

Morgan, W.

Murphy, P.

Naeem, $\mathrm{H}$.

Naerstad, R.

Negussie, E.

Nout, R.

Ochatt, S.J.

Oey, I.

Orfanoudakis, M.

Palonen, P.

Parikka, P.

Parvage, M.

Poggio, S.

Praznik, W.

Proietti, S.

Rinne, M.

Ruusunen, $\mathrm{M}$.

Savage, G.

Savin, R. 
Seljåsen, R.

Serra-Devesa, T.

Serrano, M.

Singh Malhi, S.

Skelvag, A,

Struck, C.

Timonen, $\mathrm{S}$.

Torresen, K.

Turnay, K.

Ulén, $B$.

Vanhatalo, A.

Vestberg, M.

von Cramon-Taubadel, S.

von Wright, A. 